\title{
Application of different Scheimpflug-based lens densitometry methods in phacodynamics prediction
}

This article was published in the following Dove Press journal:

Clinical Ophthalmology

6 April 2016

Number of times this article has been viewed

\author{
Fernando Faria-Correia ${ }^{1-5}$ \\ Bernardo T Lopes ${ }^{5,6}$ \\ Isaac C Ramos ${ }^{5,6}$ \\ Tiago Monteiro ${ }^{1,2}$ \\ Nuno Franqueira' \\ Renato Ambrósio Jr ${ }^{5-8}$ \\ 'Ophthalmology Department, \\ Hospital de Braga, Braga, Portugal; \\ ${ }^{2}$ Life and Health Sciences Research \\ Institute (ICVS), School of Health \\ Sciences, University of Minho, Braga, \\ Portugal; ' ICVS/3B's - PT Government \\ Associate Laboratory, Braga, Portugal; \\ ${ }^{4}$ ICVS/3B's - PT Government \\ Associate Laboratory, Guimarães, \\ Portugal; ${ }^{5}$ Rio de Janeiro Corneal \\ Tomography and Biomechanics \\ Study Group, Rio de Janeiro, Brazil; \\ ${ }^{6}$ Instituto de Olhos Renato Ambrósio, \\ Rio de Janeiro, Brazil; ${ }^{7}$ VisareRio, Rio \\ de Janeiro, Brazil; ${ }^{8}$ Department of \\ Ophthalmology and Visual Sciences, \\ Federal University of São Paulo, São \\ Paulo, Brazil
}

Purpose: To evaluate the correlations between preoperative Scheimpflug-based lens densitometry metrics and phacodynamics.

Methods: The Lens Opacities Classification System III (LOCS III) was used to grade nuclear opalescence (NO), along with different methods of lens densitometry evaluation (absolute scale from $0 \%$ to $100 \%$ ): three-dimensional (3D), linear, and region of interest (ROI) modes. Cumulative dissipated energy (CDE) and total ultrasound (US) time were recorded and correlated with the different methods of cataract grading. Significant correlations were evaluated using Pearson or Spearman correlation coefficients according to data normality.

Results: A positive correlation was detected between the NO score and the average density and the maximum density derived from the 3D mode ( $r=0.624, P<0.001 ; r=0.619, P<0.001$, respectively) and the ROI mode ( $r=0.600, P<0.001 ; r=0.642, P<0.001$, respectively). Regarding the linear mode, only the average density parameter presented a significant relationship with the NO score $(r=0.569, P<0.001)$. The $3 \mathrm{D}$-derived average density and maximum density were positively correlated with $\mathrm{CDE}$ ( $\mathrm{rho}=0.682, P<0.001$; rho $=0.683, P<0.001$, respectively) and total US time ( $\mathrm{rho}=0.631$ and $\mathrm{rho}=0.668$, respectively). There was a linear relationship between the average density and maximum density of the ROI mode and CDE (rho $=0.686, P<0.001$; rho $=0.598, P<0.001$, respectively) and total US time ( $\mathrm{rho}=0.642$ and rho $=0.644$, respectively). The average density was the only parameter derived from the linear mode that showed a significant correlation with $\mathrm{CDE}$ ( $r h o=0.522, P<0.001$ ) and total US time ( $\mathrm{rho}=0.450, P<0.001$ ).

Conclusion: Specific Scheimpflug-derived densitometric parameters of the nucleus correlated with phacoemulsification parameters. The use of the appropriate densitometry approach can predict more efficiently the phacodynamics.

Keywords: cataract, phacoemulsification, scheimpflug, densitometry

\section{Introduction}

Detailed evaluation of cataract density is essential in surgery planning in order to improve visual outcomes and to avoid possible intraoperative and postoperative complications. The Lens Opacities Classification System III (LOCS III) is a subjective evaluation method based on slit-lamp examination. ${ }^{1}$ Although this is a cost-effective grading system, interobserver and intraobserver variations influence the reproducibility of the evaluations. ${ }^{2,3}$

Anterior segment imaging has become progressively attractive with the advent of Scheimpflug-based systems, such as the Pentacam (OCULUS Optikgeräte GmbH, Wetzlar, Germany). Previous studies have enabled objective measurements of lens density, providing more precise and reliable assessments compared to the LOCS III grading system. ${ }^{4-7}$ Different approaches have been described to quantify cataract density from the Scheimpflug images, and some correlated better with lens grading and
Correspondence: Fernando Faria-Correia Life and Health Sciences Research Institute (ICVS), Universidade do Minho, Campus de Gualtar, 4710-057 Braga, Portugal

Email f.faria.correia@gmail.com (c) (7) (5) 2016 Faria-Correia et al. This work is published and licensed by Dove Medical Press Limited. The full terms of this license are available at https://www.dovepress.com/terms.php (c) ${ }_{\mathrm{BY}} \mathrm{NC}$ and incorporate the Creative Commons Attribution - Non Commercial (unported, v3.0) License (http://creativecommons.org/licenses/by-nc/3.0/). By accessing the work you hereby accept the Terms. Non-commercial uses of the work are permitted without any further permission from Dove Medical Press Limited, provided the work is properly attributed. For permission for commercial use of this work, please see paragraphs 4.2 and 5 of our Terms (https://www.dovepress.com/terms.php). 
visual performance than others. ${ }^{8-10}$ This technology has also provided the opportunity to analyze the relationship between the densitometric measurements and the phacoemulsification parameters. ${ }^{911-14}$ Concerning this topic, the variety of the evaluation and surgical methods described in the literature affects the conclusions of scientific studies. In this context, we intended to perform a comprehensive assessment of all lens densitometry methods available in Pentacam by checking their relationship with the LOCS III classification and phacodynamics in age-related nuclear cataracts.

\section{Methods}

This retrospective study was performed at Hospital de Braga, Braga, Portugal, from June to October 2014. The study enrolled patients with age-related nuclear cataract who had uneventful phacoemulsification during this period. Written informed consent was obtained from each patient before the surgical procedure. The study was approved by the Institutional Review Board (Hospital de Braga) and complied with the Declaration of Helsinki of the World Medical Association. No patient had a history of ocular disease, ocular surgery, or general disorders affecting vision, such as diabetic retinopathy or uveitis. All patients were submitted to a complete ophthalmic examination that included uncorrected and corrected visual acuity measurements, noncontact tonometry, slit-lamp biomicroscopy, and dilated fundus examination. Nuclear opalescence (NO) was assessed according to the LOCS III scale (range: 0.0 to 6.9 ) by using a slit-lamp microscope after pupil dilation with $1 \%$ topical tropicamide and $10 \%$ phenylephrine. The same ophthalmologist (FFC) performed the LOCS III evaluation. Patients with poor mydriasis, corneal opacities, cataracts with NO score exceeding 4.1, and cortical opacities were excluded from the study. Preoperative examinations also included optical biometry (ALLEGRO BioGraph; Wavelight AG, Erlangen, Germany) and Scheimpflug-based anterior segment tomography (Pentacam HR; OCULUS Optikgeräte $\mathrm{GmbH})$. In the Pentacam HR examination, the camera rotates around the eye from 0 to 180 degrees and captures 25 single slit images in less than 2 seconds. The scan collects 25,000 true elevation data points, which are processed to generate a three-dimensional (3D) representation of the anterior segment of the eye. It also provides an image of the whole crystalline lens, permitting an objective measurement of lens density. After pupil mydriasis, three consecutive scans of each eye were taken in a dark room. Regarding the lens assessment by Scheimpflug imaging, we excluded scans with artifacts that would interfere with the densitometry results, such as the presence of cortical shadowing artifacts or blinking during the scan.
We selected the Scheimpflug images on the vertical plane (90 to 270 degrees for the right eye and 270 to 90 degrees for the left eye). Three different densitometric analyses were performed to measure lens optical density on a continuous scale (Figure 1): two manual modes, ie, the linear and region of interest (ROI) modes, and one 3D mode. These densitometric methods have been described in previous studies. ${ }^{7,11}$ The software measures the lens optical density on a scale from $0 \%$ to $100 \%$ ( $0=$ no cloudiness; $100=$ completely opaque lens). The linear mode evaluates the density of a vertical line manually drawn along the axial length of the crystalline lens. The ROI mode provides a density assessment for a specific area drawn on an individual Scheimpflug image. In this study, we manually drew an elliptical ROI centered on the lens nucleus on the Scheimpflug image, excluding its anterior and posterior cortex. For the 3D mode, the software of the Pentacam system (PNS [Pentacam Nuclear Staging] software) automatically generates a cylindrical template for the density measurement. The template volume used for the study had the following features: $4.0 \mathrm{~mm}$ diameter, $2.4 \mathrm{~mm}$ height, $8.3 \mathrm{~mm}$ front curvature, and $4.8 \mathrm{~mm}$ back curvature. The $3 \mathrm{D}$ template was placed in the center of the nucleus, excluding the anterior and posterior cortex. For each densitometric mode, the average density and maximum density parameters were observed. All patients received clear corneal phacoemulsification and posterior chamber intraocular lens implantation by the same surgeon (FFC) under local anesthesia. The stop-and-chop phacoemulsification technique was performed with a Kelman microtip $0.9 \mathrm{~mm}$ 45-degree using the Infiniti System (Alcon Laboratories, Inc., Fort Worth, TX, USA). At the end of the nucleus management, the following phacoemulsification parameters were observed: cumulative dissipated energy (CDE) and total ultrasound (US) time. No intraoperative complications occurred.

All results were analyzed using Medcalc software (version 14.12.0; Ostend, Belgium) and values are reported as mean \pm standard deviation. Data normality was assessed using the Kolmogorov-Smirnov test. Significant correlations were evaluated using Pearson or Spearman correlation coefficients according to data normality. A $P$-value of 0.05 or less was considered statistically significant. However, because of the large number of comparisons ( 14 for both phacoemulsification parameters and cataract grading methods), the Bonferroni adjustment was used to reduce the possibility of a statistically significant difference based on chance alone. The level of significance was divided by the number of comparisons (14) using the Bonferroni adjustment. Thus, the $P$-value must be less than 0.004 to be considered statistically significant. 


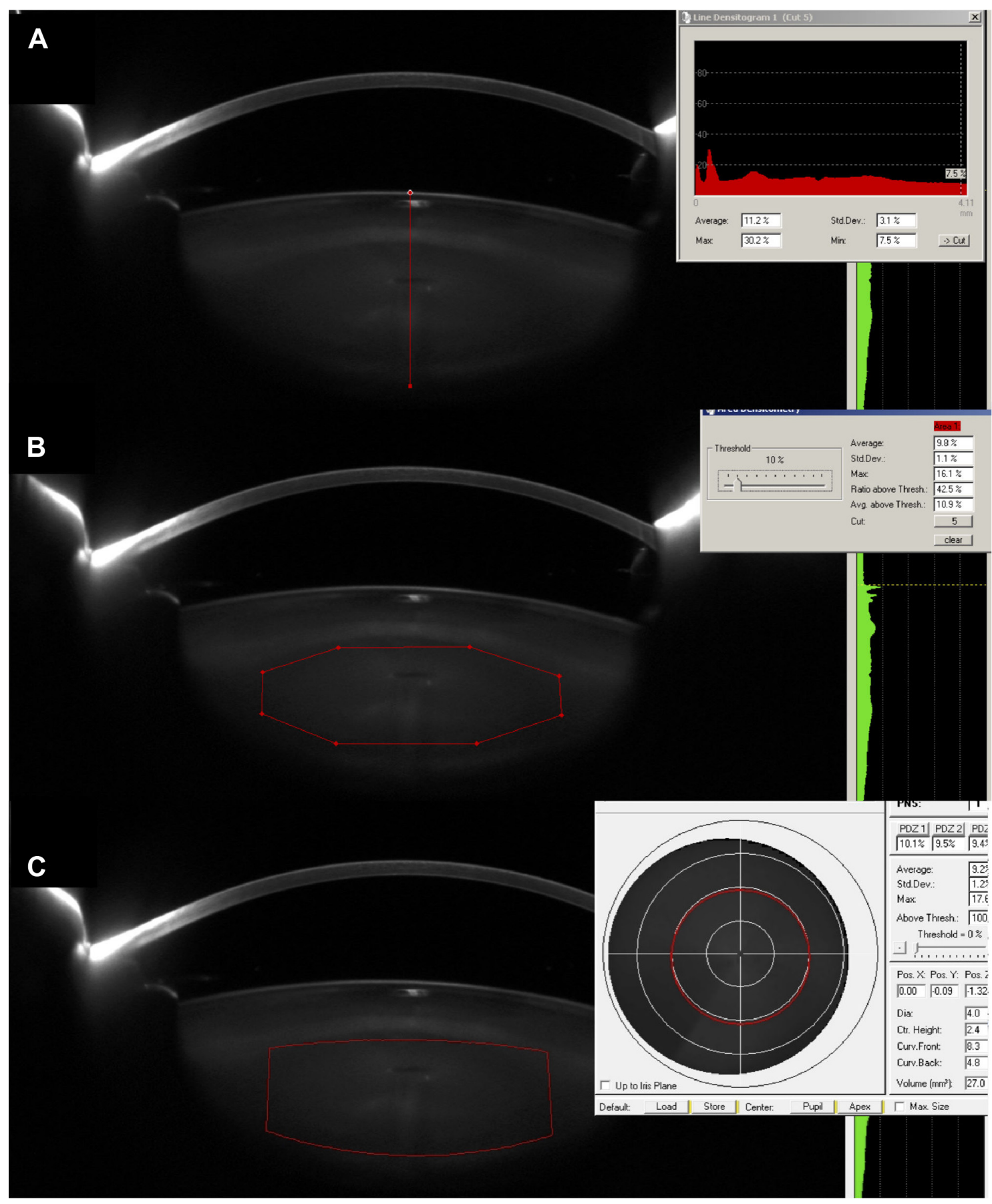

Figure I Densitometric analysis modes.

Notes: (A) Linear mode evaluates the density of a vertical line manually drawn along the axial length of the crystalline lens (red line). (B) Region of interest mode provides a density assessment inside an elliptical area manually drawn and centered on the lens nucleus (red lines). (C) Three-dimensional (3D) mode automatically generates a cylindrical template for the density measurement (red lines). The 3D template was placed in the center of the nucleus, excluding the anterior and posterior cortex.

Abbreviation: PNS, Pentacam Nuclear Staging.

\section{Results}

A stop-and-chop technique was performed in 50 eyes of 50 patients ( 30 women, 20 men) with age-related nuclear cataract. No intraoperative complications occurred. The mean age of the patients was $71.52 \pm 7.71$ years (range: 57 to 75 years). The mean NO grade was $2.88 \pm 1.02$ (range: 1.0 to 4.0 ). There were four eyes with grade 1,15 eyes with grade 2, 15 eyes with grade 3 , and 16 eyes with grade 4 . Table 1 displays the cataract evaluation by the LOCS III classification and the different Scheimpflug densitometric modes.

Table 2 shows the correlation coefficients between the LOCS III NO score and the quantification variables obtained from the different densitometric modes. There was a positive correlation between the NO score and the average 
Table I Densitometric data provided by the different evaluation methods

\begin{tabular}{lll}
\hline Cataract evaluation & Range & Mean \pm SD \\
\hline Nuclear opalescence (score) & $1.0-4.0$ & $2.88 \pm 1.02$ \\
Linear average density (\%) & $7.9-11.9$ & $9.69 \pm 0.96$ \\
Linear maximum density (\%) & $9.8-29.8$ & $17.61 \pm 4.90$ \\
ROI average density (\%) & $6.3-11.4$ & $8.44 \pm 0.87$ \\
ROI maximum density (\%) & $6.2-19.2$ & $12.11 \pm 2.63$ \\
3D average density (\%) & $6.3-11.4$ & $8.46 \pm 0.90$ \\
3D maximum density (\%) & $6.2-18.0$ & $11.97 \pm 2.23$ \\
\hline
\end{tabular}

Abbreviations: ROI, region of interest; $3 \mathrm{D}$, three-dimensional; SD, standard deviation.

density and the maximum density derived from the 3D mode ( $r=0.624, P<0.001 ; r=0.619, P<0.001$, respectively) and the ROI mode $(r=0.600, P<0.001 ; r=0.642, P<0.001$, respectively). Regarding the linear mode, only the average density parameter presented a significant relationship with the NO score $(r=0.569, P<0.001)$.

The mean CDE was $7.22 \pm 3.38 \% / \mathrm{s}$ (range: 1.78 to $20.19 \% / \mathrm{s}$ ) and the mean total US time was $40.61 \pm 19.26 \mathrm{~s}$ (range: 12.90 to $102.70 \mathrm{~s}$ ). Table 3 shows the correlation coefficients between the phacoemulsification parameters and the different evaluation methods. The densitometric parameters derived from the 3D and ROI modes showed the highest correlation coefficients with the phacoemulsification parameters $(P<0.001)$. The NO score showed only significant correlation with total US time (rho $=0.481, P<0.001$; Figure 2). The average density was the only parameter derived from the linear mode that showed a significant correlation with $\mathrm{CDE}$ (rho $=0.522, P<0.001$ ) and total US time (rho $=0.450$, $P<0.001)$. As shown in Figure 3, the 3D-derived average density and maximum density were positively correlated with $\operatorname{CDE}$ (rho $=0.682$ and rho $=0.683$, respectively) and total US time (rho $=0.631$ and rho $=0.668$, respectively). Figure 4 displays the linear relationship between average density and maximum density of the ROI mode and CDE

Table 2 Correlation coefficients between nuclear opalescence and the quantification parameters derived from the different densitometry methods

\begin{tabular}{lll}
\hline Cataract evaluation & Nuclear opalescence \\
\cline { 2 - 3 } & Correlation coefficient & P-value \\
\hline Linear average density & 0.569 & $<0.00$ I \\
Linear maximum density & $0.045^{*}$ & 0.766 \\
ROI average density & 0.600 & $<0.00$ I \\
ROI maximum density & 0.642 & $<0.00$ I \\
3D average density & 0.624 & $<0.00$ I \\
3D maximum density & 0.619 & $<0.00$ I \\
\hline
\end{tabular}

Note: *Spearman correlation coefficient.

Abbreviations: ROI, region of interest; 3D, three-dimensional.
Table 3 Spearman correlation coefficients between the phacoemulsification parameters and the different evaluation methods

\begin{tabular}{|c|c|c|c|c|}
\hline \multirow[t]{2}{*}{ Cataract evaluation } & \multicolumn{2}{|l|}{ CDE } & \multicolumn{2}{|c|}{ Total US time } \\
\hline & $\begin{array}{l}\text { Correlation } \\
\text { coefficient }\end{array}$ & $P$-value & $\begin{array}{l}\text { Correlation } \\
\text { coefficient }\end{array}$ & $P$-value \\
\hline Nuclear opalescence & 0.414 & 0.004 & 0.481 & $<0.001$ \\
\hline Linear average density & 0.522 & $<0.001$ & 0.450 & $<0.001$ \\
\hline Linear maximum density & 0.024 & $0.87 \mid$ & 0.044 & 0.722 \\
\hline ROI average density & 0.686 & $<0.001$ & 0.642 & $<0.001$ \\
\hline ROI maximum density & 0.598 & $<0.001$ & 0.644 & $<0.001$ \\
\hline $3 D$ average density & 0.682 & $<0.001$ & 0.631 & $<0.001$ \\
\hline 3D maximum density & 0.683 & $<0.001$ & 0.668 & $<0.001$ \\
\hline
\end{tabular}

Abbreviations: CDE, cumulative dissipated energy; US, ultrasound; ROI, region of interest; 3D, three-dimensional.

( rho $=0.686$ and rho $=0.598$, respectively) and total US time (rho $=0.642$ and rho $=0.644$, respectively).

\section{Discussion}

The aim of this study was to evaluate the relationship of the different lens densitometric modes available in the Pentacam system with their clinical applicability. Concerning this topic, factors that compromise the comparison of results and conclusions of previous studies are the different densitometric evaluation methods (such as PNS score, peak mode, ROI mode, and external software) and the use of different phacoemulsification techniques (such as phaco-chop and stop-and-chop). ${ }^{11-13,15}$ Although the LOCS III classification is a validated cataract grading system, there are some issues regarding interobserver and intraobserver variability. ${ }^{1,16}$ The Pentacam is a noncontact Scheimpflug system for objective imaging of the anterior segment of the eye, which provides highly reproducible measurements. This device allows objective measurements of the lens nuclear density, representing an acceptable option to the LOCS III grading system. ${ }^{8,12}$

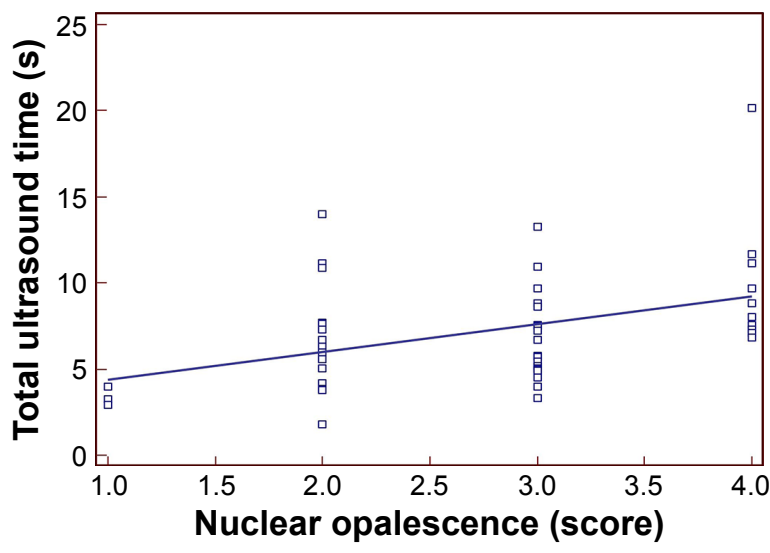

Figure 2 Relationship between total ultrasound time and nuclear opalescence. 

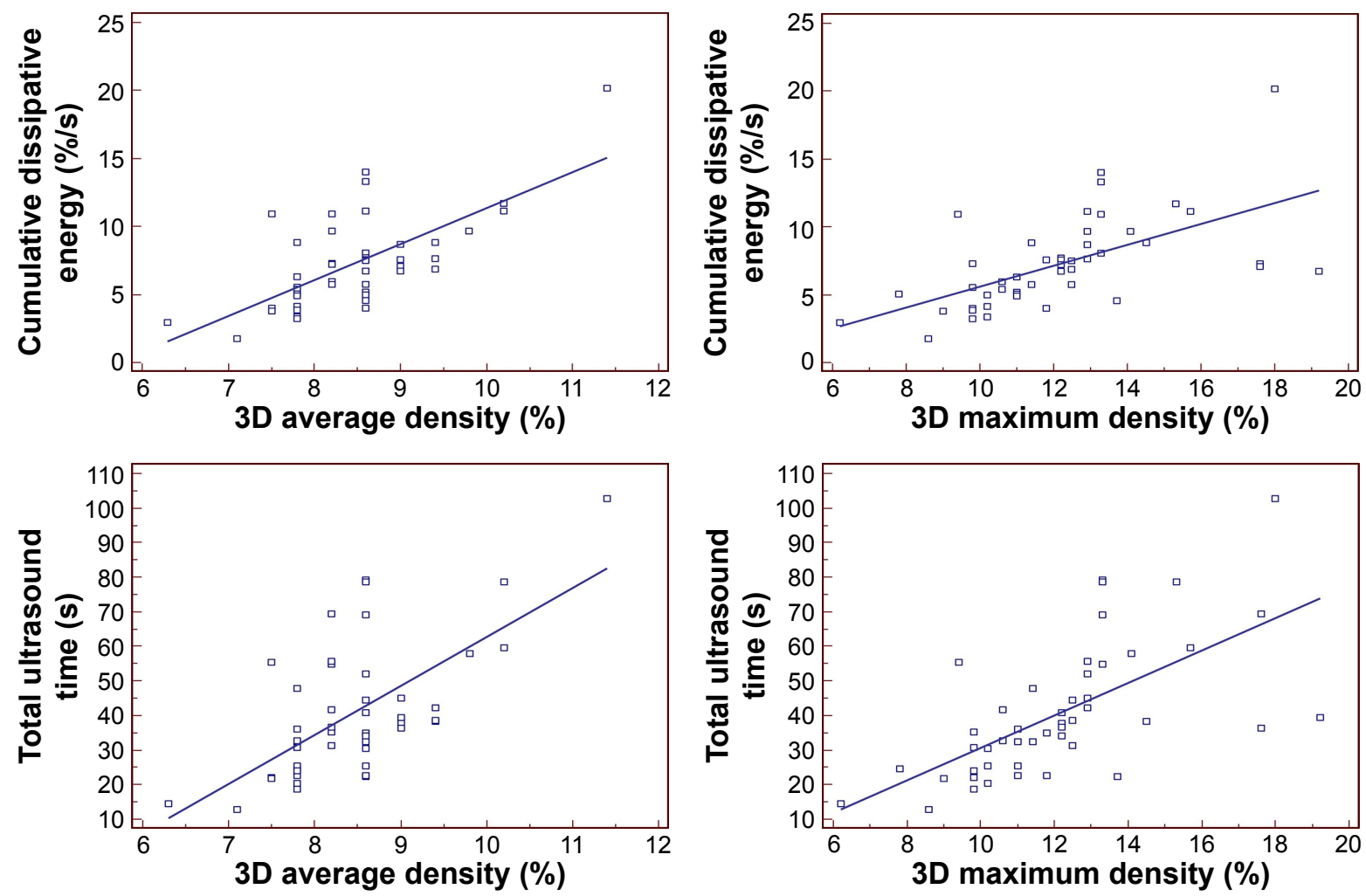

Figure 3 Relationship of average density and maximum density derived from the three-dimensional mode with cumulative dissipated energy and total ultrasound time. Abbreviation: 3D, three-dimensional.
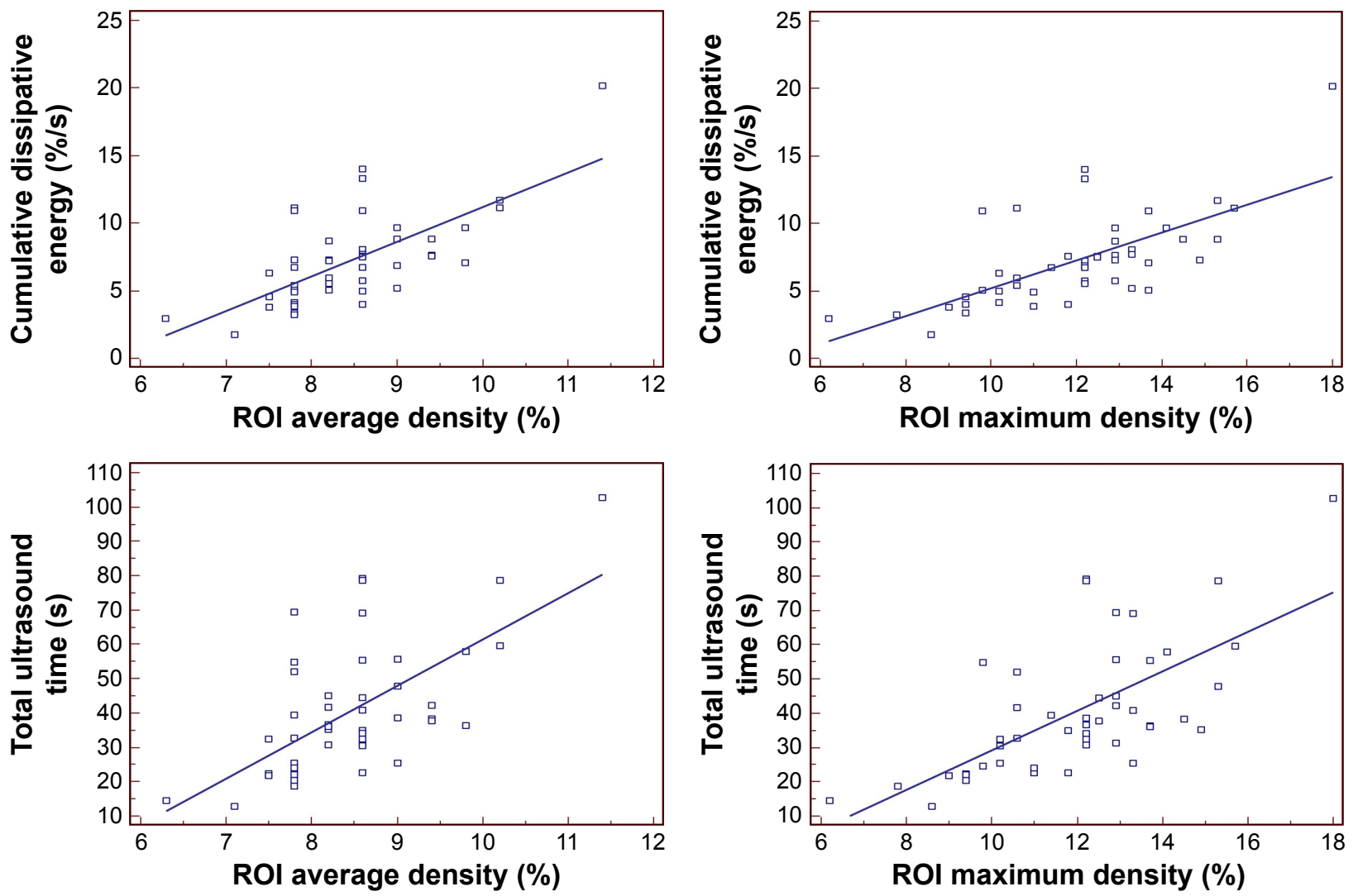

Figure 4 Relationship of average density and maximum density derived from the region of interest mode with cumulative dissipated energy and total ultrasound time. Abbreviation: ROI, region of interest. 
We need to comment about certain aspects of the methodology of the present study. Regarding the LOCS III classification, we did not consider the "nucleus color" evaluation since previous studies have reported that lens density has a stronger correlation with NO. ${ }^{10,12}$ Nuclear cataracts with an NO score higher than 4.1 were not included in the present study group. In these specific cases, the lens density is too high to allow light to pass through the nucleus and may induce erroneous densitometric values. ${ }^{7}$ Regarding the densitometry evaluation, we selected the linear, ROI, and 3D modes for analysis, which are available in the same imaging device. Compared to the PNS grading score, these methods are displayed on a continuous scale, allowing a more precise measurement of lens density., ${ }^{4,12,15}$ Although the peak mode is a method used in previous studies, we did not consider it based on repeatability reports. ${ }^{7}$ After a careful literature review, we noticed that the value provided by the peak mode basically corresponds to the maximum lens density of the linear mode used in the present study.

We found a positive correlation between the NO score and most of the nuclear density metrics, excluding the maximum density of the linear mode $(P=0.766)$. We presume that this result was due to the inclusion of the anterior cortical area of the lens in the densitometric analysis of the cited mode, which is the main region of opacification in most eyes. ${ }^{7,12}$ Another issue might be the presence of reflex artifacts in front of or within the lens. ${ }^{7}$ It is an inherent problem in this technology that may lead to higher and false maximum values.

Concerning the ROI and 3D modes, the maximum density achieved similar correlation coefficients with the NO score ( $r$ rho $=0.642$ and rho $=0.619$, respectively) compared to the average density metric (rho $=0.600$ and rho $=0.624$, respectively).

In order to increase the validity of the study, the same surgeon (FFC) performed all surgeries. "Stop-and-chop" was the selected surgical technique, since the US energy consumption for the central groove creation at the beginning of the procedure is closely related to the nuclear density. Since harder nuclei require more energy, the US energy consumption is representative of the nucleus hardness. A greater correlation was found with the metrics derived from the $3 \mathrm{D}$ and ROI modes. These findings suggest that proper selection and placement of the densitometric template may help predict phacodynamics in nuclear cataracts. We did not include the estimated balanced salt solution used in the analysis since this parameter was not correlated with Scheimpflug-measured lens density or the LOCS III score in previous studies. ${ }^{11,16}$
There are some limitations related to Scheimpflug imaging, since it is challenging to assess the posterior part of the crystalline lens, even in full mydriasis. ${ }^{810}$ Further studies with new anterior segment imaging modalities, such as anterior segment optical coherence tomography, may provide new insights into lens density measurement, allowing more precise evaluation of the opacification in specific areas. ${ }^{17}$ Other limitations of this study are the small number of cases and the exclusion of cortical and nuclear cataracts with NO score higher than 4.1 on the LOCS III classification.

\section{Conclusion}

Although having different analysis algorithms (area versus volume), the metrics of the ROI and the 3D modes presented similar correlations with LOCS III classification and phacodynamics. The present study also alerts to the fact that proper selection and placement of the densitometric template are essential for correct evaluation of nuclear cataracts. Further studies for densitometric template and metrics optimization are required.

\section{Disclosure}

Dr Ambrósio is a consultant for OCULUS Optikgeräte $\mathrm{GmbH}$ (Wetzlar, Germany), Alcon (Fort Worth, TX, USA), and Carl Zeiss Meditec (Jena, Germany). The other authors report no conflicts of interest in this work.

\section{References}

1. Chylack LT Jr, Wolfe JK, Singer DM, et al. The Lens Opacities Classification System III. The Longitudinal Study of Cataract Study Group. Arch Ophthalmol. 1993;111:831-836.

2. Karbassi M, Khu PM, Singer DM, Chylack LT Jr. Evaluation of lens opacities classification system III applied at the slitlamp. Optom Vis Sci. 1993;70:923-928.

3. Chylack LT Jr, Wolfe JK, Friend J, et al. Validation of methods for the assessment of cataract progression in the Roche European-American Anticataract Trial (REACT). Ophthalmic Epidemiol. 1995;2:59-75.

4. Kirkwood BJ, Hendicott PL, Read SA, Pesudovs K. Repeatability and validity of lens densitometry measured with Scheimpflug imaging. $J$ Cataract Refract Surg. 2009;35:1210-1215.

5. Datiles MB 3rd, Magno BV, Freidlin V. Study of nuclear cataract progression using the National Eye Institute Scheimpflug system. Br J Ophthalmol. 1995;79:527-534.

6. Magno BV, Freidlin V, Datiles MB 3rd. Reproducibility of the NEI Scheimpflug Cataract Imaging System. Invest Ophthalmol Vis Sci. 1994; 35:3078-3084.

7. Weiner X, Baumeister M, Kohnen T, Buhren J. Repeatability of lens densitometry using Scheimpflug imaging. J Cataract Refract Surg. 2014;40:756-763.

8. Pei X, Bao Y, Chen Y, Li X. Correlation of lens density measured using the Pentacam Scheimpflug system with the Lens Opacities Classification System III grading score and visual acuity in age-related nuclear cataract. Br J Ophthalmol. 2008;92:1471-1475.

9. Ortiz D, Alio JL, Ruiz-Colecha J, Oser U. Grading nuclear cataract opacity by densitometry and objective optical analysis. $J$ Cataract Refract Surg. 2008;34:1345-1352. 
10. Grewal DS, Brar GS, Grewal SP. Correlation of nuclear cataract lens density using Scheimpflug images with Lens Opacities Classification System III and visual function. Ophthalmology. 2009;116:1436-1443.

11. Kim JS, Chung SH, Joo CK. Clinical application of a Scheimpflug system for lens density measurements in phacoemulsification. J Cataract Refract Surg. 2009;35:1204-1209.

12. Gupta M, Ram J, Jain A, Sukhija J, Chaudhary M. Correlation of nuclear density using the Lens Opacity Classification System III versus Scheimpflug imaging with phacoemulsification parameters. J Cataract Refract Surg. 2013;39:1818-1823.

13. Lim SA, Hwang J, Hwang KY, Chung SH. Objective assessment of nuclear cataract: comparison of double-pass and Scheimpflug systems. J Cataract Refract Surg. 2014;40:716-721.

14. Magalhaes FP, Costa EF, Cariello AJ, Rodrigues EB, Hofling-Lima AL. Comparative analysis of the nuclear lens opalescence by the Lens Opacities Classification System III with nuclear density values provided by Oculus Pentacam: a cross-section study using Pentacam Nucleus Staging software. Arq Bras Oftalmol. 2011;74:110-113.
15. Nixon DR. Preoperative cataract grading by Scheimpflug imaging and effect on operative fluidics and phacoemulsification energy. J Cataract Refract Surg. 2010;36:242-246.

16. Davison JA, Chylack LT. Clinical application of the lens opacities classification system III in the performance of phacoemulsification. J Cataract Refract Surg. 2003;29:138-145.

17. Wong AL, Leung CK, Weinreb RN, et al. Quantitative assessment of lens opacities with anterior segment optical coherence tomography. Br J Ophthalmol. 2009;93:61-65.
Clinical Ophthalmology

\section{Publish your work in this journal}

Clinical Ophthalmology is an international, peer-reviewed journal covering all subspecialties within ophthalmology. Key topics include: Optometry; Visual science; Pharmacology and drug therapy in eye diseases; Basic Sciences; Primary and Secondary eye care; Patien Safety and Quality of Care Improvements. This journal is indexed on

Submit your manuscript here: http://www.dovepress.com/clinical-ophthalmology-journal

\section{Dovepress}

PubMed Central and CAS, and is the official journal of The Society of Clinical Ophthalmology (SCO). The manuscript management system is completely online and includes a very quick and fair peer-review system, which is all easy to use. Visit http://www.dovepress.com/ testimonials.php to read real quotes from published authors. 